\title{
SOBRE LA VALORACIÓN JURÍDICA DE LA MUERTE DE OSAMA BIN LADEN
}

\author{
Claudia Cárdenas ${ }^{1}$
}

\section{INTRODUCCIÓN}

En la conferencia de prensa que dio para anunciar la muerte de Osama Bin Laden, el presidente Barack Obama señaló que se había "hecho justicia". Mencionó también que la operación "Gerónimo", que culminó con la muerte de Bin Laden y de otras personas que vivían con él, tenía por fin capturarlo y entregarlo a la justicia ${ }^{2}$.

Con todo, informaciones posteriores a esa fecha entregan antecedentes que llevan a cuestionarse por qué, en ese caso, no fue capturado. Informaciones entregadas con posterioridad a esa conferencia inicial resultan indiciarias de que mediante la operación se pretendía más bien hacer desaparecer completa y definitivamente a Osama Bin Laden $^{3}$, como insinúa el que se tratara de una unidad militar especializada -sin haber sufrido bajas- frente a un hombre desarmado; y el hecho de que su cadáver haya sido arrojado al mar sin noticia ni participación de su familia. Esos hechos son más bien compatibles con una operación tendiente a un "homicidio selectivo" (targeted killing $)^{4}$.

1 LL.M., Dr. iur. por la Universidad Humboldt (Berlín). Profesora asociada del Departamento de Ciencias Penales de la Facultad de Derecho de la Universidad de Chile, adscrita al programa de Doctorado de la misma Facultad.

2 Cfr. http://www.youtube.com/watch?v=gPQNbG26u_0, consultada el 3 de octubre de 2011. 3 Cfr. http://www.emol.com/noticias/internacional/detalle/detallenoticias. asp?idnoticia $=479450$, consultada el 12 de octubre de 2011. Esto parece insertarse en un plan más amplio de homicidios selectivos. Lamb, Christina. Killing of al-Qa'ida leaders hurting, en The Australian, 09/19/2011, p. 12: "eight of al-Qa'ida's top leaders had been killing in recent months".

$4 \quad$ Se trata de una expresión no definida ni regulada en el derecho internacional, que se viene utilizando a partir de la política de Israel de proceder de estar forma en los territorios palestinos ocupados. Con todo, sólo un puñado de Estados los ha admitido. Más antecedentes en el estudio de Naciones Unidas sobre homicidios selectivos, en http://www2.ohchr.org/english/bodies/hrcouncil/ docs/14session/A.HRC.14.24.Add6.pdf, consultado el 3 de octubre de 2011. Hoy la expresión es 
En lo sucesivo se reseñará lo que se conoce acerca de los hechos de esa operación y se discutirán los principales aspectos jurídicos relacionados con su valoración, para finalmente volver sobre la afirmación de que "se ha hecho justicia".

\section{LOS HECHOS DE LA OPERACIÓN “GERÓNIMO”}

La operación "Gerónimo" consistió en el envío de dos helicópteros con miembros de la unidad militar especializada Navy seats a una casa en la que, según información de inteligencia, existía una posibilidad de que se encontrata Osama Bin Laden. El presidente Obama ha admitido que autorizó la operación sin tener certeza de que su objetivo se encontraba en el lugar ("[t]his is basically 50-50", "[t]his was a big gamble") $)^{5}$. Los helicópteros partieron desde Afganistán hacia la ciudad de Abotabad, en Pakistán, sin avisar a las autoridades paquistaníes.

Al llegar a su destino los militares de elite se encontraron con personas que opusieron resistencia armada a su ataque (también armado). Hubo bajas (no está claro si 5 o 6) de habitantes de la casa ${ }^{6}$ antes de que los militares llegaran hasta el piso donde estaba Osama Bin Laden. Este último habría sido llamado por su nombre por su esposa, que se encontraba con él. En definitiva se aclaró que ambos estaban desarmados, y que a ella la dejaron herida de bala en una

utilizada tanto en la prensa como en doctrina. Cfr, a saber, http://query.nytimes.com/search/sitesear ch?query=targeted+killings\&srchst=cse, consultada el 3 de octubre de 2011; y Yoo, John. Assassination or targeted killings. New York Law School Review, volume 56, 2011/12. Allí se identifica a otras personas que fueron objeto de esta práctica: Mohammed Atef (2001), Gulbuddin Hekmatyar (2002), Haitham al-Ymeni (2005), Anwar al-Awlaki (2010).

$5 \quad$ Cfr. Jefferson, David. "The day we got Bin Laden". En Newsweek 7/12/2011, vol. 158 issue 11, p. 42 y s.

6 Cfr. Rayner, Gordon, Swinford, Steven y Evans, Martin. "Day two - and anew version of bin Laden's dying moments; the contradictions the death of bin Laden". En The Daily Telegraph (London), may 4, 2011 Wednesday Edition 1: Scotland, section news, p. 4.

7 Si bien en un momento Brennan había señalado lo contrario, cfr. Rayner, Gordon, Swinford, Steven y Evans, Martin. "Day two - and anew version of bin Laden's dying moments; the contradictions the death of bin Laden". En The Daily Telegraph (London), may 4, 2011 Wednesday Edition 1: Scotland, section news, p. 4. 
pierna y a él lo mataron mediante tiros en la cabeza y el pecho ${ }^{8}$. En un primer momento se dijo que la había usado como escudo humano, luego que simplemente estaba en la misma habitación?.

Un agente de inteligencia cuya identidad se desconoce declaró que si bien Bin Laden vivía en el segundo piso de una casa que compartía con otras familias, y no contaba con teléfono ni internet, durante la operación se encontró documentación que indicaría que seguía actuando como oficial de inteligencia ${ }^{10}$.

Por su parte, John Brennan, que señaló que si hubieran tenido oportunidad de tomarlo prisionero, lo hubieran hecho ${ }^{11}$. Esa aseveración hace surgir inmediatamente la duda acerca de lo que debe entenderse por "oportunidad de tomarlo prisionero", si se nos dice que esta no existió para una unidad militar de elite ante un hombre desarmado; si esta misma unidad militar de elite optó por dispararle en la cabeza y el pecho (heridas con altísima probabilidad de ser letales) y no en una zona que permitiera su pervivencia y captura, si es que se asume que era necesario herir a un hombre desarmado para capturarlo. ¿Se esperaba acaso una ausencia absoluta de reacción ante el ataque sorpresa con helicópteros? ${ }^{32}$

No deja de ser llamativo que conforme a una encuesta Gallup el 60\% de los encuestados prefería que se matara a Bin Laden frente a la

8 Cfr. Richey, Warren. Were Navy SEALs justified in shooting Osama bin Laden?; White House Press Secretary Jay Carney announced Tuesday that Osama Bin Laden was unarmed when shot by Navy SEALs. But under the law of war, the Al Quaeda leader was a legitimate military target, say legal experts. En The Christian Science Monitor, May 3 2011, Tuesday.

9 Cfr. Rayner, Gordon, Swinford, Steven y Evans, Martin. Day two - and a new version of bin Laden's dying moments; the contradictions the death of bin Laden. En The Daily Telegraph (London), may 4, 2011 Wednesday Edition 1: Scotland, section news, p. 4.

10 Cfr. Dilanian, Ken. Death of Osama bin Laden; U.S. releases Bin Laden videos; other items gathered in the raid show he was Al Qaeda's operational leader to the end, official says. En Los Angeles Times, May 8 2011, Sunday, Home edition, main news, foreign desk, Part A, p. 10.

11 Cfr. Richey, Warren. Were Navy SEALs justified in shooting Osama bin Laden?; White House Press Secretary Jay Carney announced Tuesday that Osama Bin Laden was unarmed when shot by Navy SEALs. But under the law of war, the Al Quaeda leader was a legitimate military target, say legal experts. En The Christian Science Monitor, May 3 2011, Tuesday.

12 Carney dijo "resistant does not require a firearm". Cfr. Rayner, Gordon, Swinford, Steven y Evans, Martin. Day two - and anew version of bin Laden's dying moments; the contradictions the death of bin Laden. En The Daily Telegraph (London), may 4, 2011 Wednesday Edition 1: Scotland, section news, p. 4. 
alternativa de su captura ${ }^{13}$, y que reiteradamente se apunte a los problemas que esta hubiera conllevado ${ }^{14}$. Resulta bastante más acorde con los hechos conocidos lo afirmado por un oficial no identificado, en cuanto a que esta habría sido de una operación de homicidio ${ }^{15}$.

\section{VALORACIÓN JURÍDICA}

Dos parecen ser los puntos cruciales de cara a una valoración jurídica de la operación "Gerónimo" en general y de la muerte de Osama bin Laden en particular. De una parte, cabe examinar el argumento esgrimido por Estados Unidos en cuanto a que Osaba bin Laden era un objetivo militar legítimo; de otra, la situación respecto de la soberanía de un tercer Estado, en este caso Pakistán.

\subsection{Osama Bin LAden Como objetivo militar legítimo}

La aseveración estadounidense de que Osama Bin Laden constituía un objetivo militar legítimo presupone asumir que se está actuando en un contexto en el que es aplicable el derecho internacional humanitario, ya que es esta la rama del derecho conforme a la cual se determina la legitimidad o ilegitimidad de un objetivo militar. Además, como es sabido, es el derecho aplicable en los conflictos armados y es solamente en este contexto en el que cabe discutir la excepcional procedencia de homicidios selectivos (targeted killings) ${ }^{16}$.

13 Cfr. Richey, Warren. Were Navy SEALs justified in shooting Osama bin Laden?; White House Press Secretary Jay Carney announced Tuesday that Osama Bin Laden was unarmed when shot by Navy SEALs. But under the law of war, the Al Quaeda leader was a legitimate military target, say legal experts. En The Christian Science Monitor, May 3 2011, Tuesday.

14 Cfr. Sweet, Lynn. The mission the seals in the main paper: more on Bin Laden's death from columnists Richard Roepper, Stella Foster and Steve Huntley; daring raid took months to plan; one cornered in bedroom, terror chief used woman as a human shield. En Chicago Sun-Times, may 3 2011, Tuesday, section bulls wrapper, p. 2.

15 Cfr. Rayner, Gordon, Swinford, Steven y Evans, Martin. Day two - and a new version of bin Laden's dying moments; the contradictions the death of bin Laden. En The Daily Telegraph (London), may 4, 2011 Wednesday Edition 1: Scotland, section news, p. 4; Berkowitz, Roger. Assassinating justly: Reflections on justice and revenge in the Osama bin Laden killing. En law, culture and the humanities 2011 7:346, p. 347.

16 Si se tratara de un combatiente o de un civil que toma directamente parte en las hostilidades y el homicidio obedece a necesidades militares, proporcionado y procurando evitar daños a civiles. 
Sobre este punto, resulta pertinente tener presente que si bien las administraciones de Estados Unidos se han referido invariablemente a una guerra contra el terrorismo, más allá de estas declaraciones, para que se aplique el derecho internacional humanitario resulta necesario lo que en derecho internacional se entiende como un conflicto armado, ya sea internacional o sin carácter internacional ${ }^{17}$. Un conflicto armado es internacional si tiene lugar entre dos sujetos de derecho internacional. No es este el caso de lo que se ha dado en denominar como "guerra contra el terrorismo". Si bien alguno de sus capítulos sí han importado guerras entre Estados (a saber, Estados Unidos contra Afganistán), la denominada operación "Gerónimo", que culminara con la muerte de Bin Laden, no queda incluida en ellos. Quedaría entonces examinar la posibilidad de que esta operación haya tenido lugar en un contexto que conforme al derecho internacional importe un conflicto armado sin carácter internacional al que se aplique el derecho internacional humanitario. Este clase de conflicto de define como el que tiene lugar en el territorio de un Estado, entre sus fuerzas militares y un grupo armado o entre dos grupos armados. Queda fuera de dudas que no es este el caso de la denominada "guerra contra el terrorismo" de Estados Unidos, puesto que no cumple con ninguno de los requisitos; así, en primer lugar, no se trata de un conflicto que se desarrolle dentro de las fronteras de un Estado (Estados Unidos para el caso en comento); y en segundo término, como explica fundadamente el estudio de Naciones Unidas sobre homicidios selectivos, Al Quaeda no tiene las características que permitan comprenderlo como un "grupo armado" conforme al derecho internacional humanitario ${ }^{18}$. En la operación "Gerónimo" se enfrentaron fuerzas armadas estadounidenses a personas que vivían con Osama Bin Laden en Paquistán. Falta entonces el supuesto básico para la aplicación del derecho internacional humanitario, con

\footnotetext{
Cfr, con más antecedentes, el estudio de Naciones Unidas sobre homicidios selectivos, en http:// www2.ohchr.org/english/bodies/hrcouncil/docs/14session/A.HRC.14.24.Add6.pdf, consultado el 3 de octubre de 2011, p. 10 y s.

17 Cfr. al respecto Werle, Gerhard. Tratado de derecho penal internacional, 2da edición, Tirant lo Blanch, 2011, p. 574 y ss.

18 En http://www2.ohchr.org/english/bodies/hrcouncil/docs/14session/A.HRC.14.24.Add6.pdf, consultado el 3 de octubre de 2011, p. 17 y ss. Cfr. además Ambos, Kai y Alkatout, Josef. Der Gerechtigkeit einen Dienst erwiesen? Zur völkerrechtlichen Zulässigkeit der Tötung Osama bin Ladens, en Juristenzeitung 15/15/2011, p. 758 y ss.
} 
lo que Osama Bin Laden, en las circunstancias dadas, no puede ser considerado un objetivo militar legítimo.

En contextos diferentes de los conflictos armados, sólo cabe matar a otro de manera justificada en legítima defensa -no se ha reportado que bin Laden haya cometido una agresión contra sus captores que ameritara esa reacción, y en todo caso cabría sopesar una eventual provocación al atacar su domicilio con fuerzas armadas-, o en estado de necesidad -sin que tampoco aparezca visiblemente cual es la situación de necesidad que sólo sea superable con su muerte- o, en los Estados que así lo prevén, aplicando la pena de muerte previo proceso, lo que tampoco tuvo lugar aquí.

Así las cosas, Bin Laden no era más que alguien a quien Estados Unidos imputaba, al menos en lo que a declaraciones públicas concierne, variados crímenes. Siendo así, lo que hubiera correspondido es pedir su extradición y juzgarlo. Actuando á la Eichmann pudo pensarse incluso en secuestrarlo y juzgarlo, pero ejecutar sin más a un hombre desarmado, sin previo juicio, no parece ser una conducta ajustada a derecho respecto de la cual pueda predicarse que es un acto de justicia. Es más aun suponiendo -en contra de lo sostenido aquí- que hubiera existido un contexto de conflicto armado conforme al derecho internacional, el homicidio contra quienes han depuesto las armas es punible como crimen de guerra ${ }^{19}$, y se conoce por declaraciones de autoridades estadounidenses que Osama Bin Laden se encontraba desarmado.

\subsection{Afectación de la soberanía del tercer Estado (Pakistán)}

De las declaraciones de las autoridades estadounidenses se desprende que no se habría siquiera informado a las autoridades paquistaníes

19 Cfr. para conflictos armados internacionales, el artículo 8 pfo. 2 b) vi), y para conflictos armados sin carácter internacional, el artículo 8 pfo. 2 c) i) del Estatuto de Roma de la Corte Penal Internacional. 
de la misión, que tuvo lugar en el territorio de su Estado $^{20}$, temiendo que dicha información se filtrara hasta bin Laden ${ }^{21}$.

Así las cosas, Estados Unidos hizo uso de la fuerza armada en el territorio de otro Estado, sin siquiera informárselo esgrimiendo que la operación que culminó con la muerte de Bin Laden sería un ejercicio de legítima defensa estatal, que es una de las razones que autorizan el uso de la fuerza por los estados de conformidad con el artículo $51^{\circ}$ de la Carta de Naciones Unidas, según el cual: "Ninguna disposición de esta Carta menoscabará el derecho inmanente de legítima defensa, individual o colectiva, en caso de ataque armado contra un miembro de las Naciones Unidas, hasta tanto que el Consejo de Seguridad haya tomado las medidas necesarias para mantener la paz y la seguridad internacionales" 22 . Entiende entonces que matar a Bin Laden puede ser entendido como un ejercicio de legítima defensa.

Este argumento presenta dos falencias principales. En primer término, no sirve como argumento para invocarlo frente al Estado paquistaní. De hecho, la legítima defensa sólo puede ejercerse contra el responsable de la agresión ilegítima, y Estados Unidos no ha hecho presente que Paquistán lo hubiera agredido. En segundo lugar, el principal requisito de toda legítima defensa es la existencia presente o la inminencia de una agresión ilegítima, en este caso contra el Estado. Sería necesario explicar primero cómo es que una persona desarmada pueda representar para Estados Unidos esa clase de agresión y en qué consioste en concreto la agresión que en definitiva se evitó. Luego, debe actuarse con ánimo de defender, vale decir, el ámbito de acción sólo puede extenderse, en lo subjetivo, a hacer que una agresión actual cese o impedir que una agresión inminente

\footnotetext{
20 Cfr. Sweet, Lynn. The mission the seals in the main paper: more on Bin Laden's death from columnists Richard Roepper, Stella Foster and Steve Huntley; daring raid took months to plan; one cornered in bedroom, terror chief used woman as a human shield. En Chicago Sun-Times, may 3 2011, Tuesday, section bulls wrapper, p. 2.

21 Cfr. Rayner, Gordon, Swinford, Steven y Evans, Martin. Day two - and anew version of bin Laden's dying moments; the contradictions the death of bin Laden. En The Daily Telegraph (London), may 4, 2011 Wednesday Edition 1: Scotland, section news, p. 4.

22 Ponen de relieve que en el caso en comento el Consejo de Seguridad ya había tomado medidas Ambos, Kai y Alkatout, Josef. Der Gerechtigkeit einen Dienst erwiesen? Zur völkerrechtlichen Zulässigkeit der Tötung Osama bin Ladens, en Juristenzeitung 15/15/2011, p. 764.
} 
llegue a tener lugar. No se divisa cómo el hecho específico de la muerte de Osama Bin Laden ha servido para terminar un ataque actual o frenar uno inminente contra Estados Unidos, y en qué consistirían uno u otro $^{23}$. En el caso de Bin Laden no puede desprenderse de los hechos conocidos que haya existido un ataque actual o inminente por parte de Bin Laden, sino que más bien se presenta sólo una presunción de que él estaba en conocimiento de eventuales futuros ataques. Cierto es que Estadios Unidos ha planteado que también podría invocarse la legítima defensa como uso legítimo de la fuerza armada en los casos de amenaza permanente, pero esta posición carece de una aceptación amplia. Aun asumiendo que la documentación encontrada en el domicilio asaltado ha sido relevante para la inteligencia estadounidense, no se divisa cómo en ejercicio de fuerza armada en un tercer Estado para obtener su muerte puede caer bajo un supuesto de legítima defensa estatal. Más bien, esta argumentación parece confundir la legítima defensa del Estado con la legítima defensa como eximente de responsabilidad penal en el derecho internacional, lo que incluso el Estatuto de la Corte Penal Internacional se ocupe de distinguir claramente ${ }^{24}$. Vale decir, ni aun en el caso en que -entiéndase como ficción- pudiera afirmarse que existió o fue inminente una agresión por parte de Pakistán contra Estados Unidos que hiciera que el uso de fuerza militar en su territorio mediante la operación "Gerónimo" pudiera comprenderse como una manifestación de legítima defensa estatal, este contexto no bastaría para justificar la muerte de Osama Bin Laden y algunas de las personas que vivían con él como conductas justificadas por la legítima defensa.

Además, resulta necesario considerar que si se pretende legitimar el actuar de Estados Unidos en suelo paquistaní, habría que hacer

23 En general, el tema de los ataques llevados a cabo por agentes distintos de los Estados es discutido, cfr. el estudio de Naciones Unidas sobre homicidios selectivos, en http://www2.ohchr.org/ english/bodies/hrcouncil/docs/14session/A.HRC.14.24.Add6.pdf, consultado el 3 de octubre de 2011, p. 12 y s.

24 Artículo 31, pfo. 1 c) (dedicado a la legítima defensa como eximente de responsabilidad penal individual), oración final: "El hecho de participar en una fuerza que realizare una operación de defensa no bastará para constituir una circunstancia eximente de la responsabilidad penal de conformidad con el presente apartado". 
lo propio cuando cualquier Estado actuara en el territorio de otro alegando circunstancias equivalentes. Vale decir, sólo estimar que existe la posibilidad de que una persona a la que se imputan delitos graves de alta connotación pública se encuentra en otro Estado legitimaría para actuar con unidades militares en su territorio. Esta hipotética conclusión no parece compatible con la carta de Naciones Unidas.

\section{PALABRAS FinALES}

Sin desconocer el avance que el Talión supuso en su tiempo al limitar la venganza a la entidad de la ofensa -en esta caso, la muerte de Osama Bin Laden y algunas de las personas que vivían con él versus las muertes de las víctimas de los ataques del 11-S- no parece que la alusión a la justicia haya sido la más afortunada.

Se trata sobre todo de un problema de valoración. Teniendo a la vista la política que ha seguido Estados Unidos en la última década, no resulta sorprendente que unidades militares estadounidenses hayan dado muerte a Bin Laden. Asimismo, conociendo mínimamente las reacciones usuales en la cultura occidental, no sorprende tampoco la alegría de ciertas víctimas del 11-S ante el anuncio de su muerte. Lo preocupante son más bien dos aspectos: la consolidación de Osama Bin Laden como un mártir a ojos de sus simpatizantes, de un lado; y, de otro, el presentar en cadena no sólo nacional, sino que mundial, a una conducta que no guarda conformidad con el derecho vigente como un acto de justicia. Esa valoración puede tener algún lugar en la construcción que se ha venido utilizando para llamar en la última década a una especie de cruzada del bien contra el mal, donde, aplicando una visión agustiniana, todo ataque al mal se traduce en un bien y la conducta que lo realiza podría ser, por tanto, valorada como justa (ajustada al bien por ser contraria al mal) ${ }^{25}$. Sin embargo, no parece conveniente ni pertinente perder de vista ni abandonar 
los esfuerzos de los últimos siglos en pro de que las relaciones entre los Estados se hayan de valorar y regir conforme al derecho. El llamado es entonces a no dejarse confundir por la ya varias veces mencionada alusión a la justicia entendiéndola como equivalente a lo justo conforme a derecho, sin mayor examen. Estamos más bien ante la abierta admisión de que se ha dado muerte, sin juicio previo, no solamente a quien consideraba responsable por conductas criminales, sino a quienes a la sazón lo acompañaban; y es justamente el Estado que comandó las tropas que llevaron a cabo la operación el que valora públicamente este acto como uno de justicia.

Resulta preocupante que la reacción a la comunicación tantas veces mencionada haya sido, en su momento, de general felicitación ${ }^{26}$, encontrándose incluso "razonable" que el cuerpo de Bin Laden haya sido lanzado al mar $^{27}$. En este breve texto se ha pretendido, como contrapunto, plantear la necesidad de discutir acerca de los límites de los medios de los que disponen los Estados para actuar, aun en los casos en los que quienes cometen delitos no se han dado límites; de abordar la discusión acerca de si la ejecución sin juicio previo ha de legitimarse como solución aceptable, con todas las consecuencias que eso conlleva; o si por el contrario ha de existir alguna reacción ante la violación de ciertas normas de conducta relevantes que la comunidad de Estados ha logrado consolidar en las últimas décadas.

\footnotetext{
26 Cfr. por de pronto las reacciones del Secretario de Naciones Unidas, Ban Ki-moon, en http:// www.bbc.co.uk/news/world-us-canada-13260609, consultada el 12 de octubre de 2011.

27 Cfr. las declaraciones del Secretario General de la Organización de Estados Americanos, en http://www.cooperativa.cl/insulza-valoro-muerte-de-bin-laden-y-considero-razonable-tirar-sucuerpo-al-mar/prontus_nots/2011-05-02/205031.html, consultara el 12 de octubre de 2011.
} 